\title{
A well test model for stress-sensitive and heterogeneous reservoirs with non-uniform thicknesses
}

\author{
Zhang Liehui*, Guo Jingjing and Liu Qiguo \\ State Key Laboratory of Oil and Gas Reservoir Geology and Exploitation, Southwest Petroleum University, \\ Chengdu, Sichuan 610500, China \\ (C) China University of Petroleum (Beijing) and Springer-Verlag Berlin Heidelberg 2010
}

\begin{abstract}
In view of the anisotropy, heterogeneity and stress-sensitive permeability in low permeability reservoirs, an analytical well test model was established by introducing the concept of permeability modulus. This model considered the permeability stress-sensitivity, wellbore storage effect, and the skin effect. The perturbation technique and Laplace transformation were used to solve the mathematical model analytically in Laplace space, and the bottom-hole pressure type curves were plotted and analyzed in real space by using the Stehfest numerical inversion.
\end{abstract}

Key words: Non-uniform thickness, stress-sensitive, heterogeneous reservoir, transient well test, type curve

\section{Introduction}

Deposition heterogeneity and some measures adopted during drilling, completion and production operations in reservoirs will cause radially heterogeneous distribution of reservoir properties. In the early 1960s, Albert, Jaisson and Marion firstly proposed a well test model for two-region radial composite reservoirs (Earlougher, 1977). Loucks and Guerrero (1961) gave an integral equation to describe the pressure distribution in the composite reservoirs based on the study above. Barua and Horne (1987) proposed a well test model for three-region composite reservoirs. In the 1990s, Abbaszadeh and Kamal (1989), Bratvold and Horne (1990), and Acosta and Amabastha (1994) extended the well test model for two-region radial composite reservoirs to multi-region reservoirs and put forward a well test model for $n$-region radial composite reservoirs. Jia (1994) and Jia and $\mathrm{Li}$ (1996) proposed a well test model for multi-region radial composite oil reservoirs with variable formation thickness. Considering the fractal distribution of porosity and permeability, Xiang et al (2001) developed a mathematical model for fractal reservoirs with variable thickness and lateral heterogeneity based on fractal theory. Tian et al (2006) constructed a three-region model for radially heterogeneous, composite gas reservoirs. Later Fu et al (2006) established a well test model for multi-region, non-isopachous and laterally heterogeneous composite gas reservoirs. By extending the radial composite reservoir model to multi-region reservoirs and taking into consideration non-uniform reservoir thickness, the established well test models are more suitable for actual

*Corresponding author. email: zhangliehui@vip.163.com

Received January 1, 2010 reservoirs. However, these models do not take into account the permeability stress sensitivity, which results in deviations when applying them to predict the pressure behavior of stress-sensitive reservoirs. As more and more high-pressure and low-permeability reservoirs have been discovered, it becomes necessary to establish a well test model suitable for this type of stress-sensitive reservoirs. Based on the achievements of the predecessors, by introducing the concept of permeability modulus (Liao and Feng, 2005; Huang et al, 2007), an analytical well test model is developed for radially heterogeneous and stress-sensitive reservoirs with nonuniform thickness, which takes into account the simultaneous deformation of multi-region reservoirs. The perturbation technique (Pedrosa, 1986), Laplace transformation, and Stehfest numerical inversion method (Stehfest, 1970; Zou et al, 2007) are employed to solve the mathematical model. Type curves are calculated and plotted and the characteristics of type curves are analyzed.

\section{Mathematical model}

The mathematical model proposed in this paper is based on oil reservoir conditions; it is also applicable to gas reservoirs by replacing the pressure term with a pseudopressure term. The mathematical model is based on the following assumptions.

1) The isotropic formation can be divided into $n$ concentric regions. Each region is assumed to be uniform (i.e. the porosity and total compressibility do not change with pressure in each region) and their reservoir and fluid properties are different from each other. The reservoir thickness varies in the radial direction, as shown in Fig. 1. The greater the value of $n$ is, the closer to the actual reservoir thickness distribution the reservoir thickness $h_{j}$ is. 2) Single phase slightly compressible 
fluid and isothermal flow. 3) Laminar flow in each region and negligible gravitational and capillary effects. 4) Taking into consideration the wellbore storage effect and skin effect. 5) Stress sensitivity exists in each region and the permeability modulus of each region is the same. 6) The well is produced at a constant rate and the pressure in the whole reservoir is equal to $p_{\mathrm{i}}$ before production. 7) Assuming that the reservoir properties change abruptly at the interface and ignoring interface width and flow resistance at the interface.

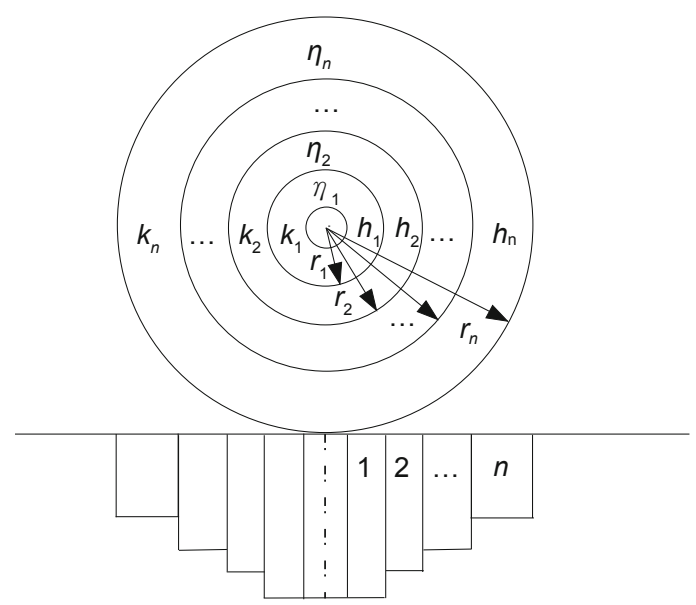

Fig. 1 Schematic of a multi-region and radially heterogeneous reservoir with non-uniform thickness

Based on the above-mentioned assumptions and basic theory of fluid flow in porous media, the following dimensionless differential equation is derived for fluid flow in multi-region, stress-sensitive composite reservoirs. This equation is based on apparent wellbore radius.

$$
\begin{aligned}
& \frac{1}{r_{\mathrm{D}}} \frac{\partial}{\partial r_{\mathrm{D}}}\left(r_{\mathrm{D}} \frac{\partial p_{j \mathrm{D}}}{\partial r_{\mathrm{D}}}\right)-\gamma_{\mathrm{D}}\left(\frac{\partial p_{j \mathrm{D}}}{\partial r_{\mathrm{D}}}\right)^{2} \quad(j=1,2, \cdots, n) \\
& =\mathrm{e}^{\gamma_{\mathrm{D}} p_{j \mathrm{D}}} \frac{1}{\eta_{\mathrm{D} j}} \frac{1}{C_{\mathrm{D}} \mathrm{e}^{2 s}} \frac{\partial p_{j \mathrm{D}}}{\partial\left(t_{\mathrm{D}} / C_{\mathrm{D}}\right)}
\end{aligned}
$$

The initial condition can be written as:

$$
p_{j \mathrm{D}}\left(r_{\mathrm{D}}, 0\right)=0 \quad(j=1,2, \cdots, n)
$$

The inner boundary conditions, which take into account the wellbore storage effect and skin effect, can be written as:

$$
\begin{aligned}
& \frac{\partial p_{\mathrm{wfD}}}{\partial\left(t_{\mathrm{D}} / C_{\mathrm{D}}\right)}-\left(r_{\mathrm{D}} \mathrm{e}^{-\gamma_{\mathrm{D}} p_{1 \mathrm{D}}} \frac{\partial p_{1 \mathrm{D}}}{\partial r_{\mathrm{D}}}\right)_{r_{\mathrm{D}}=1}=1 \\
& p_{\mathrm{wfD}}=\left.p_{1 \mathrm{D}}\right|_{r_{\mathrm{D}}=1}
\end{aligned}
$$

The infinite outer boundary condition can be written as:

$$
\lim _{r_{\mathrm{D}} \rightarrow \infty} p_{n \mathrm{D}}\left(r_{\mathrm{D}}, t_{\mathrm{D}}\right)=0
$$

There are continuous constraints in the flow interface. The pressure at the interface is:

$$
\left.p_{j \mathrm{D}}\right|_{r_{\mathrm{D}}=r_{j \mathrm{D}}}=\left.p_{(j+1) \mathrm{D}}\right|_{r_{\mathrm{D}}=r_{j \mathrm{D}}} \quad(j=1,2, \cdots, n-1)
$$

The flow rate at the interface is:

$$
\begin{gathered}
\left.\mathrm{e}^{-\gamma_{\mathrm{D}} p_{j \mathrm{D}}} \frac{\partial p_{j \mathrm{D}}}{\partial r_{\mathrm{D}}}\right|_{r_{\mathrm{D}}=r_{\mathrm{D}}}=\left.h_{\mathrm{D} j} M_{j} \mathrm{e}^{-\gamma_{\mathrm{D}} p_{(j+1) \mathrm{D}}} \frac{\partial p_{(j+1) \mathrm{D}}}{\partial r_{\mathrm{D}}}\right|_{r_{\mathrm{D}}=r_{j \mathrm{D}}} \\
(j=1,2, \cdots, n-1)
\end{gathered}
$$

The dimensionless variables involved in the above mathematical model are defined as follows:

$$
\begin{aligned}
& p_{j \mathrm{D}}=\frac{2 \pi k_{10} h_{1}}{q \mu_{1}}\left(p_{\mathrm{i}}-p_{j}\right) \\
& p_{\mathrm{wfD}}=\frac{2 \pi k_{10} h_{1}}{q \mu_{1}}\left(p_{\mathrm{i}}-p_{\mathrm{wf}}\right) \\
& r_{\mathrm{D}}=\frac{r}{r_{\mathrm{w}} \mathrm{e}^{-s}} \\
& r_{j \mathrm{D}}=\frac{r_{j}}{r_{\mathrm{w}} \mathrm{e}^{-s}} \\
& t_{\mathrm{D}}=\frac{k_{10} t}{\varphi_{1} \mu_{1} C_{t 1} r_{\mathrm{w}}^{2}} \\
& C_{\mathrm{D}}=\frac{C_{\mathrm{D}}}{2 \pi h_{1} \varphi_{1} C_{\mathrm{t} 1} r_{\mathrm{w}}^{2}} \\
& \eta_{\mathrm{D} j}=\frac{q \mu_{1}}{2 \pi k_{10} h_{1}} \gamma \\
& h_{\mathrm{D} j}=\frac{h_{j+1}}{h_{j}} \\
& k_{10} / \varphi_{1} \mu_{1} C_{\mathrm{t} 1} \\
& k_{(j+1) 0} / \mu_{j+1} / \mu_{j}
\end{aligned}
$$

where $p_{\mathrm{i}}$ is the initial reservoir pressure; $p_{j}$ is the pressure of region $j ; p_{\mathrm{wf}}$ is the bottom hole flowing pressure; $k_{10}$ is the initial permeability of region $1 ; h_{1}$ is the formation thickness of region $1 ; q$ is the sand face rate; $\mu_{1}$ is the viscosity of fluids in region $1 ; p_{j \mathrm{D}}$ is the dimensionless pressure of region $j ; p_{\mathrm{wfD}}$ is the dimensionless bottom hole flowing pressure; $\gamma$ is the permeability modulus; $\gamma_{\mathrm{D}}$ is the dimensionless permeability modulus; $r$ is the radial distance; $r_{\mathrm{D}}$ is the dimensionless radial distance; $r_{j}$ is the radius of region $j ; r_{\mathrm{w}}$ is the well radius; $r_{j \mathrm{D}}$ is the dimensionless radius of region $j ; r_{n \mathrm{D}}$ is the dimensionless 
radius of region $n ; s$ is the skin factor; $t$ is production time; $\varphi_{1}$ is the porosity of region $1 ; C_{\mathrm{t} 1}$ is the total system compressibility of region $1 ; t_{\mathrm{D}}$ is the dimensionless time; $C$ is the wellbore storage coefficient; $C_{\mathrm{D}}$ is the dimensionless wellbore storage coefficient; $h_{j+1}$ is the formation thickness of region $j+1 ; h_{j}$ is the formation thickness of region $j ; h_{\mathrm{D} j}$ is the thickness ratio; $M_{j}$ is mobility ratio; $k_{(j+1) 0}$ is the initial permeability of region $j+1 ; k_{j 0}$ is the initial permeability of region $j ; \mu_{j+1}$ is the viscosity of fluids in region $j+1 ; \mu_{j}$ is the viscosity of fluids in region $j ; \varphi_{\mathrm{j}}$ is the porosity of region $j ; C_{\mathrm{t} j}$ is the total system compressibility of region $j ; \eta_{\mathrm{D} j}$ is transmissibility factor ratio.

\section{Model solution}

The dependence of permeability on pore pressure makes the above flow differential equation group strongly nonlinear. It should first be linearized to get the analytical solution to this nonlinear equation group. We introduce the following transformation equation to linearize the equation group:

$$
p_{j \mathrm{D}}\left(r_{\mathrm{D}}, t_{\mathrm{D}}\right)=-\frac{1}{\gamma_{\mathrm{D}}} \ln \left[1-\gamma_{\mathrm{D}} \xi_{j \mathrm{D}}\left(r_{\mathrm{D}}, t_{\mathrm{D}}\right)\right]
$$

where $\xi_{j \mathrm{D}}$ is a dimensionless dependent variable; $t_{\mathrm{D}}$ is the dimensionless time.

Substituting Eq. (8) into the well test model, the perturbation technique is used to simplify the equations. Because the dimensionless permeability modulus is usually small, the zero-order perturbation solution is sufficient. Then the flow differential equation becomes:

$$
\begin{gathered}
\frac{1}{r_{\mathrm{D}}} \frac{\partial}{\partial r_{\mathrm{D}}}\left(r_{\mathrm{D}} \frac{\partial \xi_{j \mathrm{D} 0}}{\partial r_{\mathrm{D}}}\right)=\frac{1}{\eta_{\mathrm{D} j}} \frac{1}{C_{\mathrm{D}} \mathrm{e}^{2 s}} \frac{\partial \xi_{j \mathrm{D} 0}}{\partial\left(t_{\mathrm{D}} / C_{\mathrm{D}}\right)} \\
(j=1,2, \cdots, n)
\end{gathered}
$$

The initial condition and outer boundary condition are:

$$
\begin{aligned}
& \xi_{j \mathrm{D} 0}\left(r_{\mathrm{D}}, 0\right)=0 \quad(j=1,2, \cdots, n) \\
& \lim _{r_{\mathrm{D}} \rightarrow \infty} \xi_{n \mathrm{D} 0}\left(r_{\mathrm{D}}, t_{\mathrm{D}}\right)=0
\end{aligned}
$$

The inner boundary conditions which take into account the wellbore storage effect and skin effect become:

$$
\begin{aligned}
& \frac{\partial \xi_{\mathrm{wfD} 0}}{\partial\left(t_{\mathrm{D}} / C_{\mathrm{D}}\right)}-\left(r_{\mathrm{D}} \frac{\partial \xi_{1 \mathrm{D} 0}}{\partial r_{\mathrm{D}}}\right)_{r_{\mathrm{D}}=1}=1 \\
& \xi_{\mathrm{wfD} 0}=\left.\xi_{1 \mathrm{D} 0}\right|_{r_{\mathrm{D}}=1}
\end{aligned}
$$

The continuous constraints at the flow interface are:

$$
\begin{aligned}
& \left.\xi_{j \mathrm{D} 0}\right|_{r_{\mathrm{D}}=r_{j \mathrm{D}}}=\left.\xi_{(j+1) \mathrm{D} 0}\right|_{r_{\mathrm{D}}=r_{j \mathrm{D}}} \quad(j=1,2, \cdots, n-1) \\
& \left.\frac{\partial \xi_{j \mathrm{D} 0}}{\partial r_{\mathrm{D}}}\right|_{r_{\mathrm{D}}=r_{j \mathrm{D}}}=\left.h_{\mathrm{D} j} M_{j} \frac{\partial \xi_{(j+1) \mathrm{D} 0}}{\partial r_{\mathrm{D}}}\right|_{r_{\mathrm{D}}=r_{j \mathrm{D}}} \quad(j=1,2, \cdots, n-1)
\end{aligned}
$$

where $\xi_{j \mathrm{D} 0}, \xi_{(j+1) \mathrm{D} 0}, \xi_{n \mathrm{D} 0}, \xi_{1 \mathrm{D} 0}$, and $\xi_{w f \mathrm{D} 0}$ are zero-order approximations.

Combined with the initial condition (Eq. (10)), the Laplace transformation with respect to $t_{\mathrm{D}} / C_{\mathrm{D}}$ of flow differential equation in Eq. (9) yields:

$$
\begin{gathered}
\bar{\xi}_{j \mathrm{D} 0}=A_{j} I_{0}\left(r_{\mathrm{D}} \sqrt{\frac{u}{\eta_{\mathrm{D} j} C_{\mathrm{D}} \mathrm{e}^{2 s}}}\right)+B_{j} K_{0}\left(r_{\mathrm{D}} \sqrt{\frac{u}{\eta_{\mathrm{D} j} C_{\mathrm{D}} \mathrm{e}^{2 s}}}\right) \\
(j=1,2, \cdots n)
\end{gathered}
$$

where $\bar{\xi}_{j \mathrm{D} 0}$ is the Laplace transformation of $\xi_{j \mathrm{D} 0} ; u$ is the Laplace variable ; $A_{j}$ and $B_{j}$ are coefficients which can be obtained from boundary and the continuous conditions; $I_{0}$ is the zero-order modified Bessel function of the first kind; $K_{0}$ is the zero-order modified Bessel function of the second kind.

Combined with Eq. (16), the Laplace transformations with respect to $t_{\mathrm{D}} / C_{\mathrm{D}}$ of Eq. (11) through Eq. (15) yield the following $2 n$-order system of linear equations:

$$
\left[\begin{array}{ccccc}
a_{11} & a_{12} & \cdots & a_{1,2 n-1} & a_{1,2 n} \\
a_{21} & a_{22} & \cdots & a_{2,2 n-1} & a_{2,2 n} \\
\vdots & & & & \\
\vdots & & & & \\
\vdots & & & & \\
a_{2 n, 1} & a_{2 n, 2} & \cdots & a_{2 n, 2 n-1} & a_{2 n, 2 n}
\end{array}\right]\left[\begin{array}{c}
A_{1} \\
B_{1} \\
\vdots \\
B_{n-1} \\
B_{n} \\
\xi_{\mathrm{wfD} 0}
\end{array}\right]=\left[\begin{array}{c}
-1 / u \\
0 \\
\vdots \\
0 \\
0 \\
0
\end{array}\right]
$$

with

$$
\begin{aligned}
& a_{11}=\sqrt{\frac{u}{C_{\mathrm{D}} \mathrm{e}^{2 s}} I_{1}}\left(\sqrt{\frac{u}{C_{\mathrm{D}} \mathrm{e}^{2 s}}}\right) \\
& a_{12}=-\sqrt{\frac{u}{C_{\mathrm{D}} \mathrm{e}^{2 s}}} K_{1}\left(\sqrt{\frac{u}{C_{\mathrm{D}} \mathrm{e}^{2 s}}}\right) \\
& a_{1,2 n}=-u \\
& a_{21}=I_{0}\left(\sqrt{\frac{u}{C_{\mathrm{D}} \mathrm{e}^{2 s}}}\right) \\
& a_{22}=K_{0}\left(\sqrt{\frac{u}{C_{\mathrm{D}} \mathrm{e}^{2 s}}}\right) \\
& a_{2,2 n}=-1
\end{aligned}
$$

$$
\begin{aligned}
& a_{i, k}=I_{0}\left(r_{j \mathrm{D}} \sqrt{\frac{u}{\eta_{\mathrm{D} j} C_{\mathrm{D}} \mathrm{e}^{2 s}}}\right) \\
& a_{i, k+1}=K_{0}\left(r_{j \mathrm{D}} \sqrt{\frac{u}{\eta_{\mathrm{D} j} C_{\mathrm{D}} \mathrm{e}^{2 s}}}\right)
\end{aligned}
$$




$$
\begin{aligned}
& a_{i, k+2}=-I_{0}\left(r_{j \mathrm{D}} \sqrt{\frac{u}{\eta_{\mathrm{D}(j+1)} C_{\mathrm{D}} \mathrm{e}^{2 s}}}\right) \\
& a_{i, k+3}=-K_{0}\left(r_{j \mathrm{D}} \sqrt{\frac{u}{\eta_{\mathrm{D}(j+1)} C_{\mathrm{D}} \mathrm{e}^{2 s}}}\right) \\
& i=j+2 \quad k=2 j-1 \quad j=1,2, \cdots, n-2 \\
& a_{n+1,2 n-3}=I_{0}\left(r_{(n-1) \mathrm{D}} \sqrt{\frac{u}{\eta_{\mathrm{D}(n-1)} C_{\mathrm{D}} \mathrm{e}^{2 s}}}\right) \\
& a_{n+1,2 n-2}=K_{0}\left(r_{(n-1) \mathrm{D}} \sqrt{\frac{u}{\eta_{\mathrm{D}(n-1)} C_{\mathrm{D}} \mathrm{e}^{2 s}}}\right) \\
& a_{n+1,2 n-1}=-K_{0}\left(r_{(n-1) \mathrm{D}} \sqrt{\frac{u}{\eta_{\mathrm{D} n} C_{\mathrm{D}} \mathrm{e}^{2 s}}}\right) \\
& a_{i, \cdots k}=I_{1}\left(r_{j \mathrm{D}} \sqrt{\frac{u}{\eta_{\mathrm{D} j} C_{\mathrm{D}} \mathrm{e}^{2 s}}}\right) \\
& a_{i, k+1}=-K_{1}\left(r_{j \mathrm{D}} \sqrt{\frac{u}{\eta_{\mathrm{D} j} C_{\mathrm{D}} \mathrm{e}^{2 s}}}\right) \\
& a_{i, k+2}=-h_{\mathrm{D} j} M_{j} \sqrt{\frac{\eta_{\mathrm{D} j}}{\eta_{\mathrm{D}(j+1)}}} I_{1}\left(r_{j \mathrm{D}} \sqrt{\frac{u}{\eta_{\mathrm{D}(j+1)} C_{\mathrm{D}} \mathrm{e}^{2 s}}}\right) \\
& a_{i, k+3}=h_{\mathrm{D} j} M_{j} \sqrt{\frac{\eta_{\mathrm{D} j}}{\eta_{\mathrm{D}(j+1)}}} K_{1}\left(r_{j \mathrm{D}} \sqrt{\frac{u}{\eta_{\mathrm{D}(j+1)} C_{\mathrm{D}} \mathrm{e}^{2 s}}}\right) \\
& i=j+n+1 \quad k=2 j-1 \quad j=1,2, \cdots, n-2 \\
& a_{2 n, 2 n-3}=I_{1}\left(r_{(n-1) \mathrm{D}} \sqrt{\frac{u}{\eta_{\mathrm{D}(n-1)} C_{\mathrm{D}} \mathrm{e}^{2 s}}}\right) \\
& a_{2 n, 2 n-2}=-K_{1}\left(r_{(n-1) \mathrm{D}} \sqrt{\frac{u}{\eta_{\mathrm{D}(n-1)} C_{\mathrm{D}} \mathrm{e}^{2 s}}}\right) \\
& a_{2 n, 2 n-1}=h_{\mathrm{D}(n-1)} M_{n-1} \sqrt{\frac{\eta_{\mathrm{D}(n-1)}}{\eta_{\mathrm{D} n}}} K_{1}\left(r_{(n-1) \mathrm{D}} \sqrt{\frac{u}{\eta_{\mathrm{D} n} C_{\mathrm{D}} \mathrm{e}^{2 s}}}\right)
\end{aligned}
$$

The remaining coefficients are zero.

\section{Plotting and analysis of type curves}

The flow chart for plotting type curves is shown in Fig. 2.

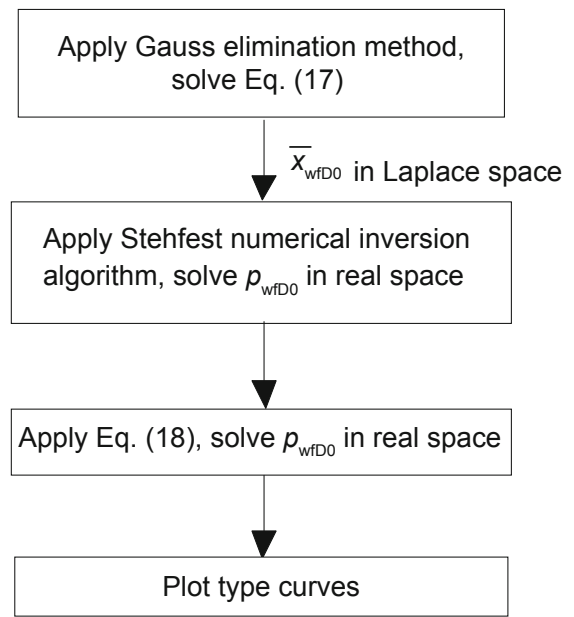

Fig. 2 Flow chart of model solving and type curve plotting

$$
p_{\mathrm{wfD}}=-\frac{1}{\gamma_{\mathrm{D}}} \ln \left\{1-\gamma_{\mathrm{D}} L^{-1}\left[\bar{\xi}_{\mathrm{wfD} 0}+O\left(\gamma_{\mathrm{D}}\right)\right]\right\}
$$

where $\bar{\xi}_{\mathrm{wfD} 0}$ is the Laplace transformation of $\xi_{\mathrm{wfD} 0} ; L^{-1}$ is the Laplace inverse transformation.

For simplicity, we plotted and analyzed the type curves for the case of $n=2$ here. However, the method proposed in this paper is applicable to the cases of multiple regions.

Figs. 3 and 4 show the type curves of radially heterogeneous and stress-sensitive reservoirs with nonuniform thicknesses. These figures show that the fluid flow in reservoirs can be divided into following several stages:

1) Wellbore storage-dominated period. During this period, fluid flow in the composite reservoir is mainly dominated by wellbore storage effect and both the dimensionless pressure and pressure derivative curves display a straight line with a slope of unity. Type curves corresponding to different dimensionless permeability modulus values are almost the same, i.e., the dimensionless permeability modulus has no effect on the shape of type curves during the wellbore storage period.

2) Transition flow period after the wellbore storage period. The duration of this period depends on the wellbore storage factor and skin factor. During this period, type curves considering or not the effect of stress sensitivity begin to separate from each other.

3) Radial flow in the inner region. The dimensionless pressure derivative curve is no longer a straight line with a slope of zero, which is the characteristic of insensitive systems. As time goes on, the departure of any $\gamma_{D}$ curve from the curve for $\gamma_{D}=0$ increases monotonically. The extent of this deviation depends on the value of the dimensionless permeability modulus. It becomes larger for greater values.

4) Transition flow period after the radial flow in the inner region. The occurrence of transient flow is related to the radius of the inner region. The greater the radius of the inner region is, the later the transient flow occurs. In addition, more significant difference in properties between the inner and outer regions (such as mobility and thickness) would lead to longer duration of this period. 
5) Radial flow in the outer region. The dimensionless pressure derivative rises (or drops) as the outer region properties become worse (or better). Similarly, the dimensionless pressure derivative curve is no longer a straight line with a slope of zero but bends upward because of the effect of permeability-stress sensitivity. The bigger the dimensionless permeability modulus is, the more significant the bend of type curves is. It should be noted that at late time, if the dimensionless permeability modulus is big enough, the pressure behavior of an infinite sensitive system is similar to that of a closed insensitive system (i.e. the pressure curve intersects with the pressure derivative curve).

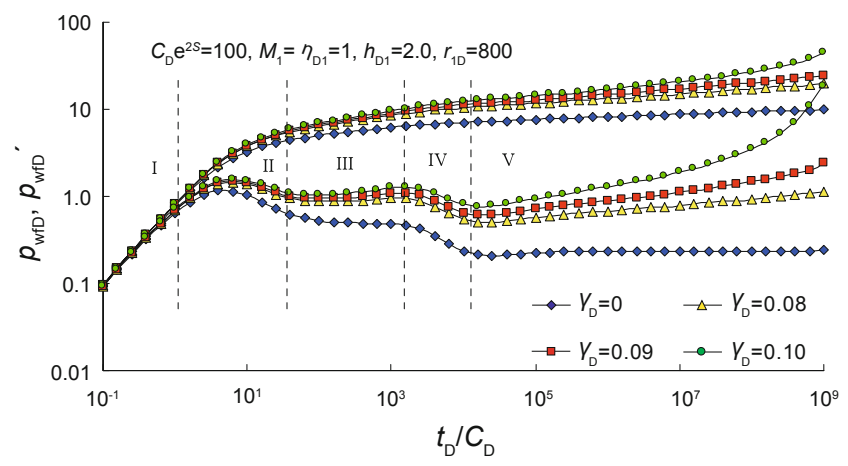

Fig. 3 Type curve of a radially heterogeneous and stress-sensitive reservoir with non-uniform thickness (Reservoir properties of the outer region are better)

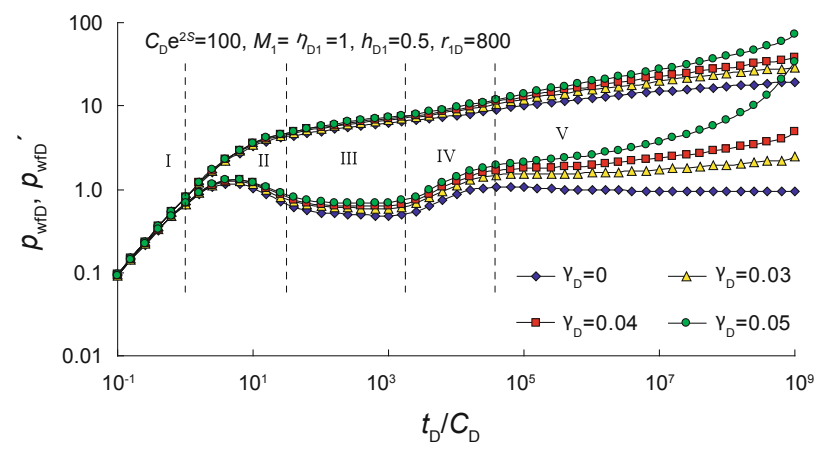

Fig. 4 Type curve of a radially heterogeneous and stress-sensitive reservoir with non-uniform thickness

(Reservoir properties of the outer region are worse)

Fig. 5 shows the effect of outer/inner region thickness ratio on the type curves of radially heterogeneous and stresssensitive reservoirs when the other parameters are constant. As Fig. 5 shows, for a given dimensionless permeability modulus, the bigger the thickness ratio is, the smaller the pressure drawdown caused by fluid flow in the reservoir is and the smaller is the impact of stress sensitivity on type curves.

\section{Model validation}

In this section, a production well is located in a strongly heterogeneous gas reservoir. Relevant parameters of the well are as follows. The well radius $r_{\mathrm{w}}$ is $0.0635 \mathrm{~m}$, reservoir

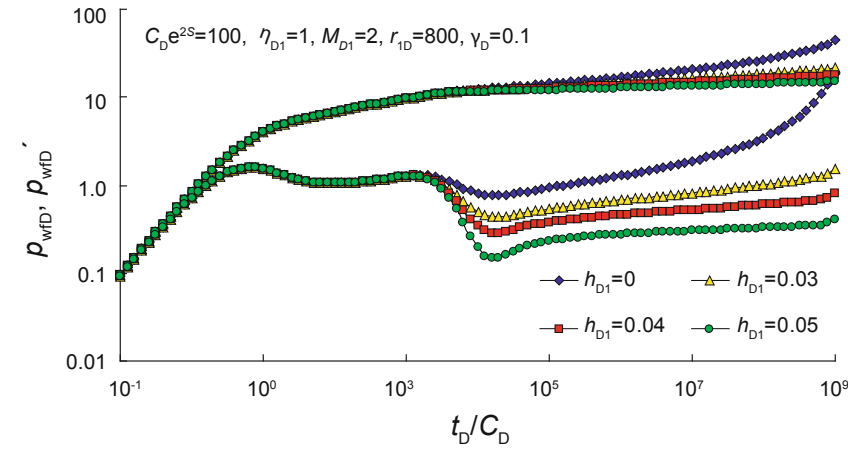

Fig. 5 The effect of thickness ratio on type curves

thickness $24.29 \mathrm{~m}$, average porosity 0.06 , and reservoir temperature is $105.9^{\circ} \mathrm{C}$. The well has produced for $3,371 \mathrm{hrs}$ at a rate of $32.3 \times 10^{4} \mathrm{~m}^{3} / \mathrm{d}$ before the pressure buildup test and the test time is $1,799 \mathrm{hrs}$.

The upward buckling of the dimensionless pressure derivative curve at the late time could not be fitted well using the conventional composite model to explain the pressure buildup data. The model established in this paper was used to explain actual well test data and satisfactory results were obtained. The log-log fitted curve is shown in Fig. 6 and the fitted results are listed in Table 1.

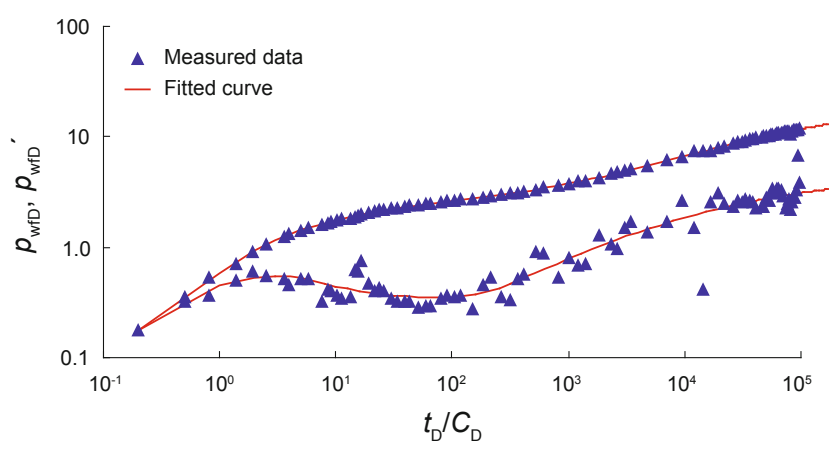

Fig. 6 Log-log fitted curves of actual well test data

Table 1 Fitted results

\begin{tabular}{cccccccc}
\hline$n$ & $\begin{array}{c}C \\
\mathrm{~m}^{3} / \mathrm{MPa}\end{array}$ & $s$ & $\begin{array}{c}k_{10} \\
\mu \mathrm{m}^{2}\end{array}$ & $\begin{array}{c}r_{1} \\
\mathrm{~m}\end{array}$ & $M_{1}$ & $\eta_{1}$ & $\gamma_{\mathrm{D}}$ \\
\hline 2 & 5.09 & -1.39 & 0.045 & 124 & 0.067 & 0.014 & 0.019 \\
\hline
\end{tabular}

\section{Conclusions}

1) A well test model for a multi-region radially composite reservoir was established, which comprehensively took into consideration the effect of stress sensitivity, wellbore storage effect and skin effect. The Perturbation technique and Laplace transformation were used to obtain the analytical solution to the model. Type curves were calculated and plotted and the characteristics of type curves were analyzed.

2) Stress sensitivity has a little impact on the bottom hole pressure behavior during the early stage. The stress sensitivity begins to affect the pressure behavior (the shape of type curves) after the wellbore storage period. The dimensionless 
pressure derivative curves during radial flow periods in both the inner and outer regions are no longer straight lines with a slope of zero but bend upward because of the permeabilitystress sensitivity. The bigger the dimensionless permeability modulus is, the more significant the extent of bend of type curves is. In addition, when the dimensionless permeability modulus is constant, the better the reservoir properties of the outer region are, the smaller the impact of stress sensitivity on type curves is.

3) The mathematical model proposed in this paper is applicable to both oil and gas reservoirs. Just by replacing the pressure term with a pseudopressure term, the model can be applied to gas reservoirs.

\section{Acknowledgements}

The authors are grateful for financial support from the National 973 Program (Grant No. 2006CB705808), PetroChina Young and Middle Aged People Innovation Fund (Grant No. 07E1016), PetroChina Science \& Technology Innovation Fund (Grant No. 2008D-5006-02-09), and Science $\&$ Technology Innovation Fund of Southwest Petroleum University (Grant No. 2007XJZ010).

\section{References}

Abbaszadeh M and Kamal M. Pressure-transient testing of waterinjection wells. SPE Reservoir Engineering. 1989. 4(1): 115-124

Acosta L G and Ambastha A K. Thermal well test analysis using an analytical multi-region composite reservoir model. Paper SPE 28422 presented at Fall Meeting of the Society of Petroleum Engineers, 2528 September 1994, New Orleans, Louisiana

Barua J and Horne R N. Computerized analysis of thermal recovery well test data. SPE Formation Evaluation. 1987. 2(4): 560-566

Bratvold R B and Horne R N. Analysis of pressure-falloff tests following cold-water injection. SPE Formation Evaluation. 1990. 5(3): 293-302

Earlougher R C. Advances in Well Test Analysis. New York: American Institute of Mining, Metallurgical and Petroleum Engineers, Inc. 1977. 97-112

Fu W S, He S L, Ran Y Z, et al. Well test analytical model and pressure characteristics of multizone, non-isopachous and lateral heterogeneous composite gas reservoirs. Oil \& Gas Geology. 2006. 27(1): 124-130 (in Chinese)

Huang Q H, Li S L, Tang H, et al. Well test analysis of gas condensate wells in low-permeable and stress-sensitive formation. Natural Gas Industry. 2007. 27(4): 95-97 (in Chinese)

Jia Y L. Well test analytical model and type curves of multizone, nonisopachous reservoirs. Well Testing. 1994. 3(4): 14-17 (in Chinese)

Jia Y L and Li Y. Well test analytical model and pressure characteristics of non-isopachous and lateral heterogeneous composite reservoirs. Well Testing. 1996. 5(3): 9-13 (in Chinese)

Liao X W and Feng J L. Well test model of stress-sensitive gas reservoirs with super-high pressure and low permeability. Natural Gas Industry. 2005. 25(2): 110-112 (in Chinese)

Loucks T L and Guerrero E T. Pressure drop in a composite reservoir. SPE Journal. 1961. 1(3): 170-176

Pedrosa O A J. Pressure transient response in stress-sensitive formations. Paper SPE 15115 presented at California Regional Meeting of the Society of Petroleum Engineers, 2-4 April 1986, Oakland, California

Stehfest H. Numerical inversion of Laplace transforms. Communications of ACM. 1970. 13(1): 47-49

Tian L, He S L and Gu D H. Effect of stress-sensitivity on well test curve characteristics in sandstone gas reservoirs. Natural Gas Geoscience. 2009. 20(2): 297-299 (in Chinese)

Xiang K L, Li Y and Li T J. A mathematical model of unstable flow of fluids through porous media with non-uniform thickness lateral heterogeneity of fractal reservoir. Petroleum Exploration and Development. 2001. 28(5): 49-52 (in Chinese)

Zou C Y, Li Z P, An X P, et al. Well test type curves plotting methods. Journal of Oil and Gas Technology. 2007. 29(3): 124-127 (in Chinese)

(Edited by Sun Yanhua) 